\title{
J
}

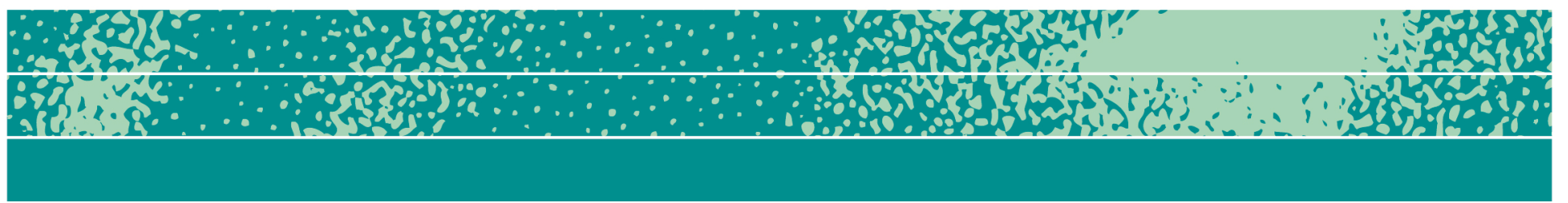

\section{Using Boundary Objects to Facilitate Culture Change and Integrate a Global Top Management Team}

\author{
Julia C. Gluesing
}

\begin{abstract}
As business anthropologists, we are often called upon to work on organizational change initiatives as members of a change team. This article is the story of one organizational change initiative involving a global top management team in a healthcare division of a large multinational firm and the research that was used as the basis for implementing change in the top management team and subsequently in the division as a whole. Specifically, the article focuses on how the change team, of which I was a part, communicated the research results to the top management team and to employees of the company by presenting the results in a map that became a boundary object, that facilitating translation across diverse groups, joint sensemaking, and local action in the change process.
\end{abstract}

\section{Keywords}

Organizational change, boundary objects, change implementation, research process

\section{Page 1 of 19}

JBA 7(1): 32-50

Spring 2018

(C) The Author(s) 2018 ISSN 2245-4217

www.cbs.dk/jba 


\section{Introduction}

As business anthropologists, we are often called upon to work on organizational change initiatives as members of a change team. In these change teams, it is common for us to assume the role of "culture expert". This role can entail providing culture general or culture specific knowledge about organizational, occupational, and national or societal culture, or it can mean investigating a specific culture or cultures involved in a change effort to help with the design, implementation, and ongoing evaluation of the change effort. In these investigations, research is generally conducted initially to assess the change context.

Anthropologists who do ethnographic work as part of the research effort can work alone or with other team members from different disciplines. However the research is conducted, there comes a point at which the research results must be communicated and used to facilitate the change effort and, hopefully, to help ensure a successful change process and outcome.

This article is about one organizational change initiative involving a global top management team in a healthcare division of a large multinational firm and the research that was used as the basis for implementing change in the top management team and subsequently in the company as a whole. Specifically, the article focuses on how the change team, of which I was a part, communicated the research results to the top management team and to the employees of the company by presenting the results as a boundary object that facilitated translation across diverse groups and sensemaking in the change process.

Before beginning the story of the organizational change initiative, I provide a brief definition of boundary objects and an introduction to their use as tools for translation and the achievement of shared meaning and understanding. The story itself starts with a description of the situation at the healthcare division when the research began, including how the change team was formed, followed by an outline of the research process to design the change solution. The story continues with how the change team developed a research results map that ultimately became a boundary object helping to create and reinforce organizational change not only in the top management team but also ultimately in the company as a whole.

\section{Boundary objects as translation and sensemaking tools}

The theory of boundary objects has been part of the anthropological tradition, especially in practicing anthropology, since Starr and Griesemer (1989) introduced the notion of the boundary object on the basis of an ethnographic study they conducted in 1989 of science teams and how they coordinated their scientific work (Trompette and Vinck 2009). Boundary objects emerge as various heterogeneous actors belonging to 
different social worlds are called upon to cooperate and to coordinate their work. Boundary objects help people with diverse perspectives create common understanding and meaning without losing the diversity of their different social worlds and the knowledge that comes with them:

The solutions invented by actors in context would seem to be of two types: the standardisation of methods and the development of boundary objects. And this concerns abstract or concrete objects, whose structure is sufficiently common to several social worlds to ensure minimum identity in terms of the intersection whilst being sufficiently flexible to adapt to the specific needs and constraints of each of these worlds. These boundary objects are supposed to maximise both the autonomy of these social worlds and communication between them. The notion is therefore closely linked to issues of shared meaning and interpretation. It supposes the existence of a minimal structure of knowledge which is recognised by the members of the different social worlds, which can take very diverse forms: the malleable object which can be shaped by each and every one; the library object from which each individual can take what he or she needs; the object which can be either simplified (abstraction), allowing us to ignore it.

(Trompette and Vinck 2009:5)

While boundary objects have different meanings in the different worlds of heterogeneous actors, different groups can recognize those meanings because they are still sufficiently structured around a common goal, e.g. to improve the functioning of a global team or to achieve a shared business objective. The notion of boundary objects is used to describe how people maintain their differences and their cooperation and how they coordinate in space and time. Thus people from different social worlds are able to negotiate differences and establish agreement on their respective points of view.

Researchers and practitioners interested in organizations and organization theory have used the theory of boundary objects to examine or address questions of design, organizational learning and knowledge management, and of particular interest to this study, organizational change. Bergman, Lyytinen, et al. (Bergman, et al. 2007) focused on ways of working using a design ecology approach, examining task, organizational and political context surrounding design. They theorized about how design products can become boundary objects that bridge functional knowledge and stakeholder power gaps across different social groups. They identified four features of design boundary objects that are essential: the capability to create shared representation, to transform design knowledge, to move people to take action and to legitimize design knowledge, all features that help align, integrate, and transform diverse technical and domain knowledge across different work groups and 
coordinate action among stakeholders who often have power gaps in organizational power. Cacciatore (2008) investigated how boundary objects can represent knowledge domains and facilitate the transfer of learning across projects. Similarly, Carlile (2002) explored how boundary objects can be a means of representing, learning about, and transforming knowledge to integrate it across functional boundaries in product development, taking a very pragmatic approach with of a view of "knowledge in practice". Levina and Vaast $(2005 ; 2006)$ studied information systems and their artifacts as boundary objects and how boundary spanning emerges as a competence among some people who are able to create a new joint field of practice that accommodates local settings and diverse interests. They draw on Bourdieu's practice theory arguing that the production of practices often involves their embodiment in objects. Boundary objects provide an opportunity to develop new shared conceptions of activity and new ways of behaving and are at the heart of organizational transformation (Macpherson and Jones 2008).

In the case I describe in this article, there was no original intention to create a boundary object to facilitate change in the global top management. However, after the research was conducted, new conceptions of the global team emerged among its members around the "situational map" that our research team created to communicate results. The practice of making sense of the data we had gathered in our research embedded our knowledge of what we thought as consultants would help the global team. We aimed to help the team transform itself from a collection of individuals working on various aspects of an organizational goal to a team with members who had common knowledge and understanding of their current situation and who could develop together their shared transformational goal and new practices over time to achieve it. The next section of this article details the situation faced by the global team before the members began their transformational work.

\section{The Situation}

A large German company, hereafter called The Company, decided it wanted to get into the emerging market for home healthcare based on digital technologies. The Company already had a division in another part of Europe that was in the emergency response end of the business. That division created home technologies, for example, that enabled the elderly or homebound chronically ill to call an emergency team for help just by pushing a button. The company acquired two competing companies in the United States that were based on both coasts and that had complementary technologies using internet-based communication to share patients' vital signs and health status with physicians and other care providers, and set up an office in London that was responsible primarily for marketing the company's products. A marketing office was 
also established in Germany. The west coast office became the company headquarters led by a German CEO and a team of experts who were responsible for developing the primary home health device that would incorporate the best of the merged organization's technologies using sound, clinically based patient health data.

The CEO was an MD who had practiced in a hospital setting doing both clinical work and research. He left this practice to assume a role as a consultant in the health care area for a large global firm. He had a very successful 10-year career as a healthcare consultant and wanted to try his hand at running a company. He did not have a business background, other than what he had picked up in his consulting role, but he had confidence in his ability to lead, especially in the healthcare industry where he had considerable expertise and had advised other companies about how to become profitable or develop an organizational structure and processes that would contribute to their success.

When the opportunity to join The Company arose, he took it. When he was officially on board, he lobbied for the merging of the emergency response business with the newly formed Healthcare Division, and with reluctance, The Company agreed to his proposal because they had confidence in his expertise. Over his first few months as CEO, he created a management team of about 18 top experts in sales, technology development, clinical research, manufacturing, and finance, and appointed one of his former associates from the consulting firm as his chief of staff and operations officer. The team included people who had been leaders in the acquired companies that had been merged to form the new Healthcare Division and brought in others from the outside. Most of the companies employees in product development were based on the west coast where the new Division's primary technology offering had been developed and would continue to be based. The technology had been created with venture capital money and had been quite successful in getting large long-term contracts, so The Company thought it had the best potential to lead the Division's growth.

However, after only 18 months of operation, the CEO realized that his top management team was floundering. The Company called into question the Division's direction because it was not producing the projected or expected results in the marketplace. The CEO was hearing rumors about employee dissatisfaction, too, and had a human resources survey conducted to get feedback from employees around the globe. $\mathrm{He}$ was surprised to learn that people did not have confidence in the Division's leadership. Morale was low. The CEO was aware of discontent among the experts on his leadership team as well. In particular, there was tension between the technology lead, who was a lead innovator of the technology offering in the Palo Alto company before it was acquired, and the clinical lead, who was an MD consultant based in another country. The rift cascaded throughout the rest of the organization and led to an "us 
versus them" mentality between the technology group and the clinical group in the Division. The sales people around in Europe and the U.S. were not cooperating well with each other either. The CEO also knew that many on the leadership team were questioning the role of his Chief of Staff as well as the role of the U.S. marketing lead, a German executive who was brought in to lead the U.S. sales effort. The CEO decided that a change at the top was necessary to transform both the direction the business was headed as well as the overall culture of the company to become more positive, cooperative, and synergistic.

The CEO hired a training and consulting firm that The Company has employed successfully in the past to help in similar situations. The consulting firm created a three-person team, of which I was a part, to work with the CEO on the creation of a change plan, beginning with the global top management team.

\section{Developing the change solution}

The change solution process was co-designed by the CEO and our consulting team using two approaches as a guide: transformational leadership (Bass and Riggio 2006; Bass 1999)and global teaming process principles (Gluesing 1998; Gluesing, et al. 2003; Gluesing and Gibson 2004). In an assessment about six months prior to our consulting engagement, a human resources consulting and assessment firm conducted a survey with all the employees in the Healthcare Division to assess morale and the perceptions employees had of the effectiveness of their leaders. The results were not encouraging. Employees believed the leadership team did not provide adequate direction or inspire employees to contribute. They thought the Division's mission was unclear and that the leadership team members were often in conflict with one another. Therefore, when the Division hired our consulting group to help improve the performance of the leadership team, they asked that one of our team members be an expert in transformation leadership. Transformational leaders inspire employees to look beyond their own self-interest for the good of the company and achieve these results by providing vision and a sense of mission that instill pride and gains respect and trust. Based on the findings of a series of surveys and on clinical and case evidence, Bass (1999) has determined that transformational leaders are good at communication their expectations, and they use symbols to focus employees efforts and communicate their desires. They also promote careful problem solving through rational consideration and treat employees as individuals, coaching and advising employees to use their intelligence

The results of the human resources assessment also indicated that the leadership team had a lack of alignment, causing employees confusion. Employees also did not believe the top management team 
members were available to them to advise when they encountered conflicting directives coming from different leaders. To address these issues, our consulting team drew on research about designing and forming global teams for effectiveness. Gluesing and Gibson (2004)

Determined in a review of research on global teaming processes that no matter what the type of global team an organization creates, the complexity the team faces in meeting its objective can be characterized along five different dimensions: task, context, people, time and technology. They discuss these five dimensions and how they interact with one another to contribute to complexity in global teams and provide a series of suggested actions for designing global teams to help manage complexity. Our consulting team drew on these suggestions in working with the Division to design a change solution for the top management team.

The change process was to be implemented in a three-phase process: Phase 1: An assessment of the current situation, Phase 2: A leadership workshop to develop an action plan based on the assessment results, and Phase 3: Ongoing coaching of the leadership team in the implementation of the action plan.

The Phase 1 assessment involved multiple methods, both qualitative and quantitative. In other words, it was a mixed methods, ethnographic approach to get as broad and holistic a picture as possible of the Healthcare Division's current situation in order to make informed recommendations to the leadership team about how to proceed with a cultural change plan. We began by examining company documents that described the expertise of the leadership team and their backgrounds, presentations given by the CEO at "all hands meetings", and other documents that might offer clues about varying cultures or management approaches that were being merged together to create the Division. We also created an ethnographic history along a timeline to portray major events that might have had an influence on the current state.

Next, we began a series of informal conversations with the CEO and his Chief of Staff, and they introduced us to the entire leadership team. The introductions took place in face-to-face and virtual meetings, because the leadership team of eighteen people was globally dispersed in various European locations, the U.S., Canada, and South America, meeting only once a quarter in face-to-face sessions. Our research team sat in on weekly meetings over a three-month period. I was usually in the meetings on the west coast with the CEO and the leaders based at the headquarters office, and my colleagues participated virtually. One of my research colleagues and I also participated in the leadership meetings from Germany. WebEx was the communication technology preferred by the leadership team, but collaboration was limited to audio and document sharing; there was no video conferencing. We learned quite a lot about 
the team dynamics by watching and listening to their interactions, paying attention especially to the decision-making process because complaints about the lack of decision-making or leadership direction were prevalent in the employee survey feedback and in the conversations with the members of the leadership team. We transcribed our field notes of the meetings so that we could share and analyze them.

While we were proceeding with the document analysis and the ongoing observations, we conducted about 30 interviews with all eighteen of the leadership team members and many of their direct reports to get a better idea of people's perceptions of the challenges facing the leadership team and to learn their ideas about what needed to be done to turn things around. The interviews were conducted in the U.S. and in Europe and almost all of them were face-to-face. We interviewed one team member via Skype and two via phone. Each interview lasted about an hour and half. We had the interviews transcribed for analysis.

In addition to the observations and the interviews, we conducted an online global teaming survey to assess people's understanding of the leadership team's mission and objectives, the team's communication processes, including the use of technology, the characteristics of the members and nature of the relationships among the team members, and the characteristics of the team's working contexts or work locations. The survey included both closed and open-ended questions and was administered company-wide with a response rate of $72 \%$.

\section{The Research results “Map" becomes a boundary object}

Our consulting team gathered all the documents, interview transcripts, observation notes, and survey data together in a qualitative data analysis software package. I took the lead on the analysis, but all three of us on the team worked together over a period of three days to make sense of the results and to compile our research report for presentation to the leadership team and conclude our Phase I assessment, which had taken approximately four months. It was during this sensemaking process that the three of us created a network map of our research results. We followed a Grounded Theory (Straus and Corbin 1990) approach, working inductively in analyzing the data, examining conditions, action strategies, and consequences. I had begun the map and presented it to my other two research team members. Then together, based on the coded interview data, document analysis, and notes from our observations, a systems view of the current state of the Division emerged. We had our boundary object, and the first notion of how we might use this map not only to communicate our research results to the top management team but also how the map might serve as a sensemaking device for the team in our Phase 2 Workshop. At the start of our research process and even in the analysis and interpretation of the data, we did not set out intentionally to 
create the map. It began as a rough starting point for me to transfer what I had learned in analyzing the data to my other two team members. As we worked together, we realized how useful the map was to our sensemaking and especially as an emerging embodiment of our interpretations and development of shared understanding across our disciplinary boundaries.

Etienne Wenger (2000)in his work on communities of practice specifies that the notion of boundary object can be broken down into four dimensions:

1. Abstraction: it facilitates dialogue between worlds

2. Multi-tasking: several activities or practices are possible

3. Modularity: different parts of the object can serve as a basis for dialogue between actors

4. Standardization of the information contained in the object: rendering the information interpretable.

The map of the Healthcare Division's current state was a boundary object as defined by Wenger. It was an abstraction of the relationships we found in analyzing the data and could help us facilitate a workshop that would span the social worlds of the top management team members, as it had done for our consulting team. Starr and Greisemer (1989) called this type of map an ideal type that does not specifically describe the details of any one locality or thing, but one that is abstracted from all domains and that works for everyone because they can translate it into relevant practices in their own social worlds. We could envision using the map in different ways, breaking it into three modules that could serve as the starting point for different groups in our planned workshop. The information contained in the map was standardized and reflected the shared terminology and language we had heard our interviewees use as they described their experiences at the Division and with the top management team. It believed it would be easy for everyone involved to interpret the information contained in the map and use it in determining the actions needed to change the way the top management team had been working.

Figure 1 is the full map ${ }^{1}$ that our consulting team presented to the top management team in our Phase 2 Workshop, designed to communicate the research results and work together with the members of the team to develop an action plan to improve their performance and that of the Healthcare Division as a whole. We used a PowerPoint version of the map at the CEO's suggestion and to conform to the standardized format for their quarterly meetings, while preserving the nature of our

\footnotetext{
${ }^{1}$ The map excludes any information that could identify the Division or its employees to preserve confidentiality.
} 
ethnographic approach and grounded theory methodology.

\section{Map of The Healthcare Division's Situation}

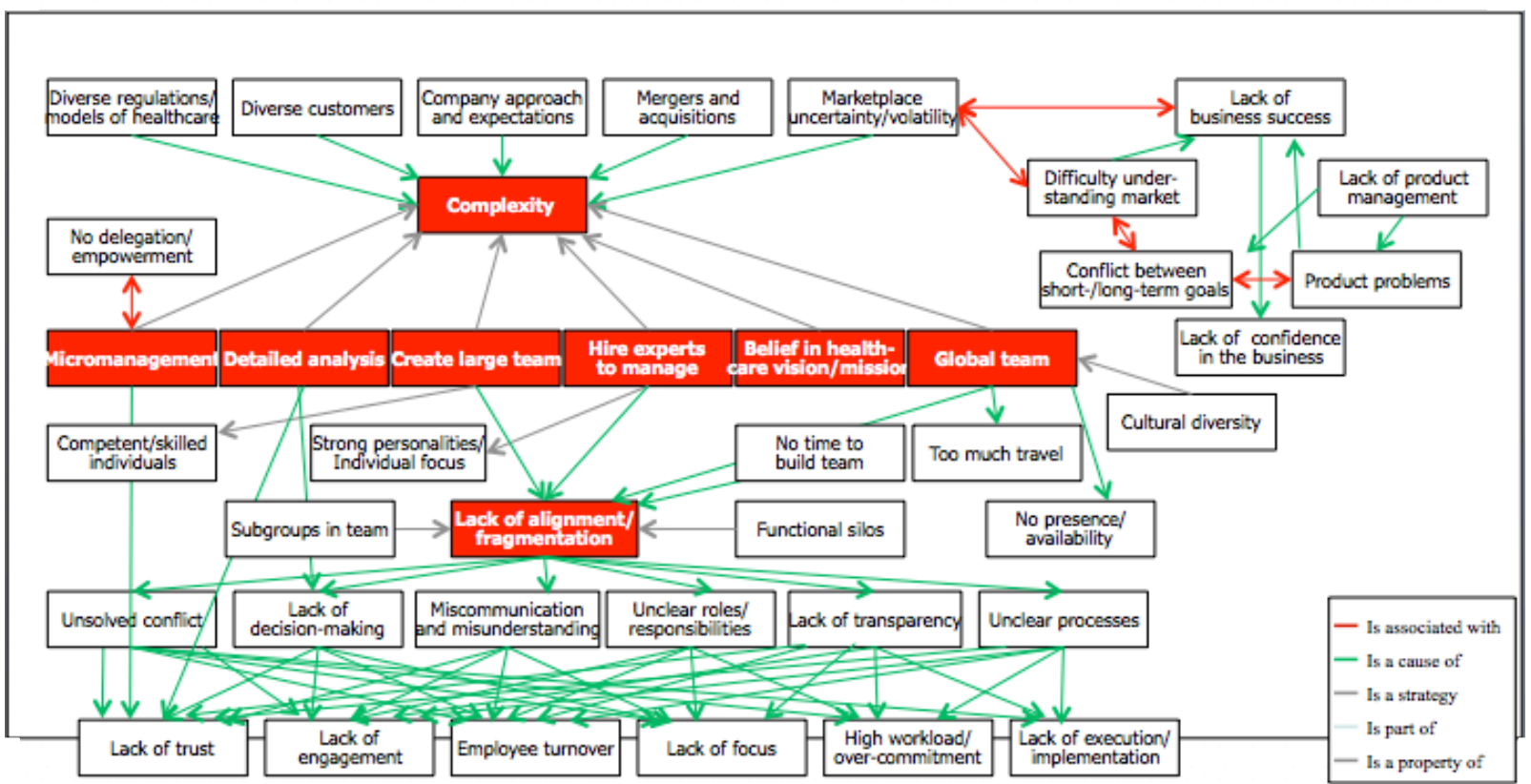

Figure 1: The Map of the Healthcare Division's Situation depicts the results of the research conducted in Phase I of the change process and became a boundary object for shared sensemaking and developing actions for change to improve performance.

The map provides an overview of the Division's situation from an ecological perspective showing how the conditions of complexity in the healthcare technology industry context were enacted by the top management team, and how their actions to cope with their situation created a lack of alignment among the top management team members which then led to low employee morale and a host of undesirable consequences. The map depicts three distinct modules: 1 ) the complexity of the healthcare technology industry; 2) the strategies undertaken by the global top management team to cope with this complexity; 3) and the consequences of these strategies for the top management team and the organization as a whole. At the bottom right of the map is a legend that explains the color-coding of the arrows depicting conditions, action strategies, and consequences. Our consulting team presented an interpretation of the map to the top management team:

1) Complexity - Diverse regulations and operating models for delivery of services, particularly those involving communication technologies that facilitated interaction between providers and patients, characterized the healthcare marketplace in which the Division was embedded. The Division was developing 
healthcare communication technologies in multiple countries, each with its own unique requirements. In addition, the company served a diverse base of customers, ranging from governmental agencies to clinicians in private practice. The Division's progressive approach and Silicon Valley-like preference for rapid innovation and few rules were often at odds with the expectations and norms of The Company, which was well established with standardized process and procedures everyone was required to follow. The Company created Healthcare Division through a series of mergers and acquisitions of existing smaller companies, some of them previous competitors, and each of them with company cultures that were very different. The diverse ways of working inside the Division and norms for interacting with the marketplace contributed to the complexity. There was also a great deal of marketplace uncertainty and volatility because the healthcare communication technology industry was relatively new and there were many entrants in the industry with no dominant product design. Because of this uncertainty and continual flux, the top management team had difficulty understanding the market, which led to a lack of business success. There was conflict in the team between short- and long-term goals and a resulting lack of product management, which in turn led to product problems and an ultimate lack of confidence in the business.

2) Global Top Management Team Strategies - To address the complexity in the marketplace, the global top management team, under the direction of the CEO, engaged in several action strategies, most of which had proven to be ineffective. When the CEO came on board he recruited a group of experts from different disciplines and regions of the world to form his global top management team. They represented finance, information technology, clinical medicine, marketing, and operations in multiple countries. Each team member had substantial experience and a strong, positive reputation in his or her respective fields of expertise. The CEO, because the company was fairly new, adopted a hands-on management style and micromanaged the top management team. He also required his team members to do detailed analysis prior to making any decisions. The team was large, 18 people, because the CEO felt he needed to have all the expertise on the team to stay on top of the volatile marketplace. All members of the global team strongly believed in the healthcare vision and mission of the Division, which was a driving force behind their desire to find a better way to work together as a team and improve the performance of the Division. 
3) Lack of Alignment/Fragmentation - While the strategies adopted by the top management team to achieve success in the marketplace made sense to the CEO and his team members, they had unanticipated negative consequences. There was a great deal of cultural diversity among the team members, both national and occupational, and because of the volatility and resultant uncertainty in the marketplace, the team members were constantly traveling and had no time to engage in any team building to develop alignment and shared understanding of the Division's vision and mission. The frequent travels also meant the managers often were unavailable to employees for direction and problem solving. The expertise among the team members as competent and skilled individuals was invaluable in building the quality products, but it also came with a downside. The team members all had strong personalities and a focus on their own personal success to maintain their reputations. The large size of the team also led to the formation of subgroups in the team and functional silos. Consequently, there was unresolved conflict among the top management team members, a lack of decision-making, frequent miscommunication and misunderstanding, unclear roles and responsibilities, a lack of transparency and unclear processes for employees to follow. Among employees morale was understandably low. They had a lack of trust in management and lack of engagement in their work. Employee turnover was high. Employees had a high workload and were often overcommitted on projects, but at the same time there was a lack of focus and lack of execution and implementation due to the unclear processes and poor and often conflicting direction for their managers.

It is not difficult to understand why the Division believed they were in trouble and that the top management team needed to change if they were to achieve success in the marketplace. There were many questions and comments about the map. The most striking among them was a comment from the director of engineering who exclaimed that it was the first time he had a full picture of what was going on in the Division and why. Other team members agreed, saying they all knew about their own piece of the Division but did not have this system's view and understanding of the connections between their actions and the consequences for both the global top management team and the Division. In all the team's virtual work, in their regular WebEx meetings, and quarterly face-to-face meetings, they had been so focused on location reports, on solving immediate problems related to one group or another, mired in details and faced with deadlines, that they had never put all the pieces together to form an overall picture of the organization's situation. Unexpectedly, no one questioned the validity of what they had heard. My 
belief is that they all could see themselves in the map and where able to learn how they all fit into to the big picture We supported our presentation of the map with quotes from the interviews and data from the survey, too, which helped reinforce the credibility of the map.

Following the presentation of these research results, the next step was to facilitate sensemaking among the top management team members to create shared understanding of their situation that would move them to action.

\section{Facilitating sensemaking}

The Phase 1 Workshop took place over two and half days, with all 18 of the top management team members present at the regular quarterly offsite meeting location in Palo Alto, California. The workshop was one part of their offsite meeting. After our three-person team introduced the map in a PowerPoint presentation with an open question and answer session, we divided the top management team into four working groups and charged them with making sense of the map themselves and developing actions to address the issues as they saw them.

We used the map as a boundary object to facilitate discussion and sensemaking among the working groups, giving each of them a two-foot by four-foot laminated poster of the map that they placed on an easel and could examine closely and even write notes on as they discussed what they had learned. The group work was lively, and our research team moved from group to group, answering questions of clarification about the results and the process, but also pushing the groups to come up with what the map meant to them. The map as boundary object produced a fluidity in the exchange of interpretations and meanings characteristic of the diverse ways of knowing that were part of the management team members experiences; it provided opportunities to use inclusive practices to facilitate deliberation (Feldman, et al. 2006).

At the end of the first day, each group reported on their progress and the whole top team management team discussed what they heard and learned from each report. Our research team took notes and worked into the night to merge and organize the groups' work into a consolidated interpretation of the map.

\section{Achieving shared understanding and gaining commitment}

The second workshop day began again with a PowerPoint presentation, this time with the groups' consolidated interpretation of the map. Nothing in the map had changed. The groups had simply provided detail and agreement about what they had understood the map to mean for them in their work. Following the presentation, our research team provided a copy of the consolidated interpretation to each member of the 
top management team. As a whole, we spent the first couple of hours coming to shared understanding and agreement about the interpretation and prioritizing the issues to be addressed. Then we moved on to developing specific actions, this time breaking up the whole team into groups based on a matching of team members' expertise and interest with the issues. By the end of the day, each team member had taken ownership of specific actions and committed to delivering by the next face-to-face meeting of the top management team.

It was difficult for the team members to accept their personal responsibility for the situation in which the Division found itself. However, the map made it easier to see that the problems were systemic and not the fault of any one of them individually. They were able to come to terms with what they needed to do to remedy the situation, and the workshop itself was a team building exercise. The team members had a chance to voice their own perspectives, based on their own social worlds and respective ways of knowing, and to be heard by the others. The deliberations and emerging agreements about the situation produced renewed commitment by the team members to working together. In fact, on the concluding half-day of the workshop, the energy was high and the entire leadership team signed a document of commitment to the recommendations they had agreed upon as a group and made a video of themselves all pronouncing individually and as a whole team "I will change," and "We will change". This video was posted on the Division's internal website for all employees to see.

\section{Reinforcing change}

It was important as part of the change process to take advantage of the momentum achieved in the workshop and extend it to achieve the goals the top management team had set out for themselves. We continued our work with the team over the next nine months to reinforce the change process by working to help the leadership team members become true change agents and by refining their skills to lead change. However, as an outside consulting team, we did not have ultimate control over the change process.

\section{Developing change agents}

Following the workshop, we worked with the CEO and various members of the top management team to prepare them for what would be required of them when communicating to the Division's employees. They then traveled to the Division's different locations with the laminated maps and their action plans to explain to the rest of the organization what they understood to be the current situation and what they were going to do about it to bring about positive change. They solicited additional input 
from employees at each location. Feedback from the leadership team was encouraging. We learned that the maps continued to serve as boundary objects to achieve shared understanding of the Division's situation. Employees felt their concerns had been heard and that something was going to change for the better in the top management team

\section{Refining skills to lead change}

In addition to the traveling presentations, the CEO proceeded with Phase 2 of the change process, the 360 leadership assessments and the individual coaching for members of the leadership team in transformational leadership. Our consulting team administered leadership assessments with everyone on the leadership team and scheduled one-hour individual coaching sessions based on the results. The leadership assessments were met positively, but they ultimately did not have as much impact as we thought they would because the leadership team changed dramatically in the next few months.

Phase 3 of our change process turned out to be not the ongoing coaching we anticipated but another workshop, this time in Europe, to discuss the progress of the actions the team had decided to implement earlier and to make some announcements about changes in the leadership team. Our consulting team was asked once again to facilitate this process.

One of the recommendations our consulting team had made (we kept our own recommendations to just four or five because we wanted the team to develop their own and take responsibility for them) was to limit the size of the top management team to a core of five people to reduce the complexity of their teamwork. The team decided to take us up on this recommendation, and several people on the team would no longer be part of the top management team going forward. All, even those who were not going to be part of the core team, accepted this announcement eagerly just to be done with the contention and the difficulties of working to manage the complexities of both a large global team and an uncertain marketplace.

This last workshop, nine months after the start of our culture change project began the end of our engagement with the Division. There were a few more informal chats with the CEO and some of the members of the team, but the leadership team was on their own in implementing change after the workshop. It is difficult to assess how well the change process has gone since our consulting team disengaged and the Board of Directors and The Company took many of the subsequent actions, out of our purview or influence. However, we do know that the CEO, his Chief of Staff, and the engineering technology lead are no longer with the Division. The Company had challenged the Division at the time we began our engagement to produce results or be disbanded. Since the Division is still 
in business, something must have gone right for them. It is difficult to know what has led to the turnaround, but I suspect that removing the CEO who had a tendency to micromanage, the elimination of the contentious relationship between the engineering lead and the clinical lead, and the creation of a much smaller and manageable leadership team who could take decisions much more rapidly and clearly in the face of flux in the marketplace, have contributed to the Division's continuation.

\section{Concluding thoughts}

While the map as a boundary object originated as a co-creation among the three of us in the multi-disciplinary consulting team as a way to commonly understand our research results and present them, it also served the same kind of sensemaking function among the diverse social worlds and localities that comprised the Division through a process of discovery and analysis during the workshop. It was a risk to introduce our map of the current situation as boundary object, because it was created by outside consultants and not arrived at by the members of the top management team. It could have easily been rejected. It is my belief that because we took an ethnographic approach in conducting the research and created an emic representation in the map itself using the language of the community, the team members felt they also participated in the co-creation of the boundary object. During the two-day workshop they were effectively able to see a unifying picture of their organization that also depicted their diverse perspectives about their difficult situation. They made the map their own. The members belonging to different social worlds were able then to use this picture to create a set of actions tailored to their different locations but consistent with a shared understanding of the situation and a common goal that they themselves had arrive at and that cut across organizational boundaries. What originated as a representation of research results became a boundary object for the top management team and also for the organization as the team members took the map to their own localities. It was a standardized way of achieving a common picture of the organization's situation and creating localized actions to improve it.

What could we as a consulting team have done differently to further the implementation of change? Not much, in my opinion. Because we were outside consultants, we could not take ownership of the change effort. We could only provide insight and facilitation. The engagement and the ownership had to come from the people in the top management team. They did take ownership of the assessment results and plan some actions to alter the current situation to move it toward the type of company and culture they desired. Fluctuations outside the Division, including imperatives presented by The Company as parent, took the team in different directions beyond our control, and even that of the top 
management team. The CEO, although he was a healthcare expert, was probably the wrong person for the job. He and The Company eventually recognized that, and he left the Division. Change did happen, and some of the major recommendations we had made did get implemented, primarily the reduction in size of the leadership team. There is only so much outside consultants can do to create change. They must work through the members of the organization, and there are always many factors outside the control of everyone involved in a change process. Perhaps we need to consider what success means and how it should be judged in varying situations. Full implementation of a change plan is not necessarily the best measure of success.

I consider the change process itself to be a success, as far as we could take it, particularly in our discovery and use of the situation map as a boundary object. It worked well for us as our consulting team made sense of the research results. Our consulting team worked well together and had very positive relationships with everyone on the top management team using a co-creation approach. The workshops themselves, and the map as boundary object, helped the leadership team developed a shared understanding of the current situation and frame of reference for creating actions to change it and accountability for these actions. Serving as facilitators more than as consultants with the answers contributed to the cooperative relationship we shared with our client. The assessment process and the workshops were all developed collaboratively with the CEO and varying members of the leadership team, particularly the Chief of Staff. Being honest about what we learned in the assessment was critical, as was the CEO's and the leadership team's acceptance of the results. Without that, we could not have moved forward with any of actions. The team's commitment to their mission was also an important aspect of change implementation. The team members truly believed in what they were doing and were committed to making the Division work. Engagement like this is key to successfully implementing change.

There are only two alternative approaches that I believe we might have employed in our own consulting effort. First, it would have been helpful to learn more about The Company's view. All our information was second hand, filtered through the CEO and others on the team, particularly the engineering lead. Second, the team badly needed some training in global teaming processes. They could have used a permanent facilitator. The Chief of Staff had assumed this role, but he was not particularly good at it. We might have pushed more for this training in hindsight. Nonetheless, the leadership team was indeed too large, creating unnecessary complexity. If we could have stayed on as consultants following the reduction in team size, it would have been worthwhile to do some global team training and facilitation, especially around strategies for managing complexity. 


\section{References}

Bass, Bernard M, and Ronald E Riggio 2006 Transformational leadership: Psychology Press.

Bass, Bernard M. 1999 Two Decades of Research and Development in Transformational Leadership. European journal of work and organizational psychology 8(1):9-32. https://doi.org/10.1080/135943299398410

Bergman, M., K. Lyytinen, and G. Mark 2007 Boundary objects in design: An ecological view of design artifacts. Journal of the Association for Information Systems 8(11):546-568. https://doi.org/10.17705/1jais.00144

Cacciatori, E. 2008 Memory objects in project environments: Storing, retrieving and adapting learning in project-based firms. Research Policy 37(9):1591-1601. https://doi.org/10.1016/i.respol.2008.04.028

Carlile, P. R. 2002 A pragmatic view of knowledge and boundaries: Boundary objects in new product development. Organization Science 13(4):442-455. https://doi.org/10.1287/orsc.13.4.442.2953

Feldman, M. S., et al. 2006 Ways of knowing and inclusive management practices. Public Administration Review 66:89-99. https://doi.org/10.1111/j.1540-6210.2006.00669.x

Gluesing, Julia 1998 Building connections and balancing power in global teams: toward a reconceptualization of culture as composite.

Anthropology of Work Review 18(2):18-30. https://doi.org/10.1525/awr.1998.18.2-3.18

Gluesing, Julia, et al. 2003 The development of global virtual teams. In Virtual teams that work: creating conditions for virtual team effectiveness. C.B. Gibson and S. Cohen, eds. Pp. 353-380. San Francisco: Jossey-Bass.

Gluesing, Julia C., and C. Gibson 2004 Designing and Forming Global Teams. In Handbook of Research on International Organizing and Managing. M.L.M. In H. Lane, M. Mendenhall ed. Pp. 199-226. Oxford: Blackwell.

Levina, N., and E. Vaast 2005 The emergence of boundary spanning competence in practice. implications for implementation and use of information systems. Mis Quarterly 29(2):335-363.

https://doi.org/10.2307/25148682

Levina, N., and E. Vaast 2006 Turning a community into a market: A practice perspective on information technology use in boundary spanning. Journal of Management Information Systems 22(4):13-37. https://doi.org/10.2753/MIS0742-1222220402 
Macpherson, A., and O. Jones 2008 Object-mediated learning and strategic renewal in a mature organization. Management Learning 39(2):177-201. https://doi.org/10.1177/1350507607087580

Starr, S., and J. Griesemer 1989 Instutional ecology, translations and boundary objects: Amateurs and professionals on Berkeley's museum of vertebrate zoologie. Social Studies of Science 19(3):387-420.

Straus, Anselm, and Juliet Corbin 1990 Basics of qualitative research. Grounded theory procedures and techniques.

Trompette, Pascale, and Dominique Vinck 2009 Revisiting the notion of boundary object. Revue d'anthropologie des connaissances 3(1):3-25.

Wenger, Etienne 2000 Communities of practice and social learning systems. Organization 7(2):225-246.

https://doi.org/10.1177/135050840072002

Julia Gluesing is a business and organizational anthropologist with more than 40 years of experience in industry and academia as a consultant, researcher and trainer in global business development focusing on global leadership development, managing global teams, managing change, innovating across cultures and cross-cultural communication and training. Julia is a part-time faculty member in the Industrial and Systems Engineering Department at Wayne State University where she teaches the management of technology change and serves as a leadership project advisor in the Engineering Management Masters Program. She also teaches courses in qualitative methods, global leadership, and global perspectives in the Global Executive Track Ph.D. Program, for which she was a founder and codirector. Julia has extensive research expertise as the principal or coprincipal investigator on five National Science Foundation grants. She can be reached at j.gluesing@wayne.edu. 